\title{
ROBUST K-NEAREST NEIGHBOR CLASSIFIER AND NEAREST MEAN CLASSIFIER BASED INFORMATIVE KNOWLEDGE DISTILLATION FACE RECOGNITION
}

\author{
M.Shanmuganathan ${ }^{1}$, T.Nalini ${ }^{2}$ \\ ${ }^{1}$ Research Scholar, Dept of Computer Science and Engineering (C.S.E), Bharath Institute of Higher Education and \\ Research(BIHER), Chennai-600073 Tamilnadu, India. \\ ${ }^{2}$ Professor, Dept of Computer Science and Engineering, Dr. M.G.R. Educational and Research Institute, Chennai-600095 \\ Tamilnadu, India. \\ Ishanmca75@gmail.com,2drnalinichidambaram@gmail.com
}

\begin{abstract}
Human Face identification procedures have made tremendous progress in the last decade. Nevertheless, identifying faces with incomplete impediment is as yet challenging for the present face identifiers, and is very much required within certifiable application programs regarding reconnaissance and protection. Though great examination exertion has been dedicated to creating face de-impediment techniques, the greater part of them can just function admirably under obliged conditions. In this manuscript is proposed a Robust K-NNC (K-Nearest Neighbor Classifier) and NMC(Nearest Mean Classifier ) (RKNNC-NMC) prototype to efficiently reestablish incompletely occluded faces even in nature. This model comprises of two-stream, first introduced to perceive high-resolution faces and goal corrupted appearances with a student stream and a teacher stream, separately. A Teacher stream is signified by a Complex RKNNC-NMC for the sake of highexactness recognition, and the student stream is signified by an a lot more straightforward RKNNC-NMC for lowunpredictability recognition. Broad examinations on synthetic and real datasets datasets of countenances with impediment plainly show the viability of RKNNC-NMC in eliminating various kinds of impediment in one's face at different locations. The suggested technique additionally gives better behaviour gain than other de-occlusion strategies in advancing recognition execution through partially-occluded faces.
\end{abstract}

Keywords: Face recognition,k-NNC(Nearest neighbor classifier and NMC-Nearest mean classifier, Feature matching and Template matching .

\section{Introduction}

Human face identification takes quite a long time being a troublesome procedure. Over the most recent twenty years, specialists have made strenuous efforts to overcome this difficulty and have obtained some acceptable outcomes. A portion of these past endeavors was centered around face recognition[1][2][3]. Pentland and Turk [3] effectively utilized an Eigen face towards dealing with perceiving a person's face. Notwithstanding, a precise as well as productive strategy for person face discovery is as yet deficient. Govindaraju et al.[4][5] introduced a framework that helps find a person's appearances in the photos; however, a rough size as well as the expected variety of countenances shall be known ahead of time. Sirohey[6] used a circular framework to extract individual heads from jumbled photos. Huang along with Yang[7] used a three tier progressive information to find individual appearances in complicated foundations. Poggio along with Sung[8][9][10]utilized two separation measurements to gauge the separation between the information image and the gathering place. Group of twelve groups including six non-face and six face bunches have been prepared utilizing an adjusted k-mean grouping strategy. A component vector comprising of 12 qualities was contribution to the multi-layer perceptron network for the sake of task checking. Pentland et al.[11][12][13][14][15][16] applied head part analysis(Principal Component Analysis) to portray human face designs with less dimensioning highlights. Whoever planned a separation work named good ways based on highlight space(dffs) as a measurement to assess a contrast between a piece of information and individual image along with a reproduced person's image. A framework might be viewed as an exceptional instance of Sung as well as Poggio's framework. Face detection can likewise be accomplished by identifying mathematical connections among facial segments, for example, the eyes, the nose, and the mouth. Juell along with Marsh[17] suggested a various leveled neural organization to identify person countenances in blurred scale footages. An edge upgrading pre-processor along with four back propagation neural organizations was orchestrated into a progressive structure conceived to discovering different countenances in an arena. Leung et al.,.[18][19][20] 
consolidated a lot of nearby element locators by means of a measurable model to discover human facial segments for face human face finding. Their own methodology was unvarying concerning interpretation, revolution, and scale. Likewise, they could deal with fractional impediment of appearances. Yet, for face recognition issue, inferable from the non-linear and complex circulation of face images under a detectable variety in perspective, enlightenment or outward appearance, the straight methods, for example, PCA or LDA, cannot give dependable and powerful answers for those face recognition issues with complex face varieties [21].

\section{Proposed System}

In this manuscript is proposed Subject Independent Automatic Facial Expression Recognition from Partially Occluded Still Footages by utilizing Appearance Based Feature Extraction Schemes. The fundamental target of this work is to build up a method that perceives face utilizing RKNNC-NMC. In this system methodology of the refining cycle comprises of about three phases. From the main stage, instate the Teacher along with the Student stream to perceive higher-goal human faces along with their low-goal variants, separately. Within the subsequent phase, this method specifically extracts just the utmost useful in the face highlights from the Teacher stream by tackling a meager chart improvement issue. In the third phase, the chosen highlights are utilized to normalize the tweaking cycle of the Student stream utilizing KNNC-NMC. Thus, the Student streams are for perceiving low-resolution human individual faces in the wild which is really prepared through at the same time taking care of two errands with restricted computational assets: particular element guess and low- resolution face distinguishing proof to recognize impeded and expressive appearances
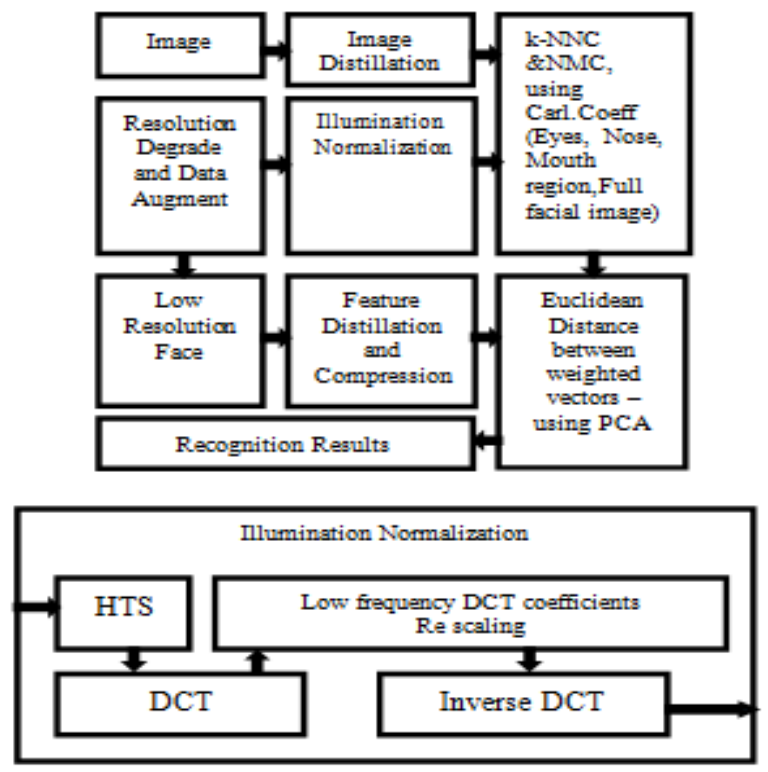

Fig. 1. Proposed System architecture - Robust k-NNC(kNearest Neighbor Classifier) and NMC(Nearest Mean Classifier) based on Informative Knowledge Distillation

\section{Working Principle}

One of the most standard non-parametric systems is a closest neighbor request (NNC), that exactly communicates that an unclassified thing comes allotted to a class for NN (nearest neighbor) amongst a ton of setup objects. NNC asymptotic or boundless model size botch is not as much as twice the Bayes botch. Various varieties of NNC are given in the composition. In the present utilization, k-NNC ( k-nearest neighbor classifier ) and NMC (Nearest Mean Classifier) have been used. This helps us to accept or characterize the key proportions from a two-stream KNN-NMCs as follows

\section{Teacher stream:}

An educator stream $\varphi \mathrm{t}(\mathrm{F} ; \mathrm{Wt})$ is the complex $\mathrm{KNNC}$ with the arrangement of boundaries $\mathrm{Wt}$ pre-prepared for perceiving a high resolution human face $(\mathrm{F})$. Herein we accept that $\mathrm{Wt}$ retains the abundant information encoded within huge highgoal human face footages from an educator face data set Dt, in what way each human face is marked by a number from the personality set Lt.

\section{Student stream}

The Student stream $\varphi s\left(\mathrm{~F}^{\sim}\right.$; Ws $)$ is a lot more straightforward KNNC for perceiving a lower-goal face $\mathrm{F}^{\sim}$ within boundaries Ws. That is found out from that understudy face set Ds $=\{(\mathrm{Fi}$ , $\left.\left.\left\{\mathrm{F}^{\sim} \mathrm{ij}\right\} \mathrm{N} \mathrm{j}=1, \mathrm{li}\right)\right\}|\mathrm{Ds}| \mathrm{i}=1$, where $|\mathrm{Ds}|$ is the quantity of high goal faces.

\section{Discriminative Knowledge Extraction from a Teacher Stream}

After an introduction phase, the student stream for the most part experiences low recognition exactness over lowresolution human faces since numerous personality signals are absent throughout the goal debasement. Accordingly, its boundaries should be adjusted again under the oversight of the educator stream to figure out how to extricate the most discriminative highlights in any event, when the face resolution is lower.

\section{Teacher-supervised Student Stream Fine-tuning}

Despite choosing instructive appearances, their own highlights removed by this Teacher stream, a tweaking of the student stream will mutually tackle two issues:

1) Estimating the highlights of informational faces presented by the Teacher stream by means of highlight relapse

2) Reclaiming the disappearing facial prompts from lowergoal faces. In this way, we might adjust an understudy stream through taking care of the minimization issue minimum Ws, where the impacts of the characterization misfortune and the relapse misfortune are joined along with equivalent significance to deal with various tasks learning issues.

\section{Experimental result analysis}

To exhibit the viability of the recommended prototype assess it upon two impeded facial datasets, which contain real and synthesized occlusion. In this manuscript, represent subjective after effects of occlusion elimination in addition to quantifiable assessment on human face recognition. 
A. Datasets 1) Training (Learning)Data: As it is difficult to gather adequate impeded countenances and the relating impediment free ones, all things considered, to display blocked appearances into the wild and learn(train) the prototype on an integrated dataset from the CASIA(Chinese Academy of Sciences)-WebFace dataset. CASIA-WebFace which contains 494,414 face images and 10,575 subjects slithered from the Internet. Altogether selecting approximately 380000 close frontal countenances $\left(-45^{\circ} \sim+45^{\circ}\right)$ from the entire data set along with blend impediment brought about by 9 kinds of basic items on these appearances. The impeding articles we use incorporate glasses, shades, covers, eye veils, hands, scarfs, telephones, cups,books etc. Every single kind of impeding item has one hundred distinct layouts, away from which $50 \%$ are utilized in favour of creating impediment on preparing information and the remaining are utilized for testing information. For every face, we haphazardly choose one layout upon 9 kinds of impediment to produce blocked face. Several impediment formats need a right area, for example, shades, glasses and veils. The proposed model include these layouts onto explicit areas of the appearances concerning identified facial tourist spots. Different formats are included onto arbitrary areas of the countenances to upgrade variety of the created information. Entire face images are edited as well as coarsely adjusted by 3 central issues situated during the focuses of mouth and eyes, and afterward resized to $128 \times 128 \mathrm{dim}$ phase ones. Figure 4 outlines a few instances of blocked appearances produced utilizing this methodology.

2) Test Data: This experiment utilize Low-Resolution Face Verification (LFW) datasets for testing. The LFW set of data includes a sum of 13,233 face images of 5,749 subjects, which were gathered from the Internet.

Results and Comparisons 1) Occlusion Removal: In this experiment initially mien into intermediate outputs of the proposed Robust K-NNC and NMC throughout the treatment of occlusion elimination, which are visualized in Figure 3.

It tends to be seen that our prototype eliminates impediment bit by bit. In particular, toward the initial phase, the human face remaking organization of the prototype creates a plausible profile of the facial image, where impeded segments might not be so clear as non-blocked segments. An impediment expectation structure gives a rough assessment of an impediment area. At that point the yields are refined continuously upon the conditions of past advances. To confirm the capacity of our recommended model for impediment evacuation, here represent subjective correlations within a few strategies including Principal Component Analysis (PCA), LPP and UDP with various kinds of impediment.
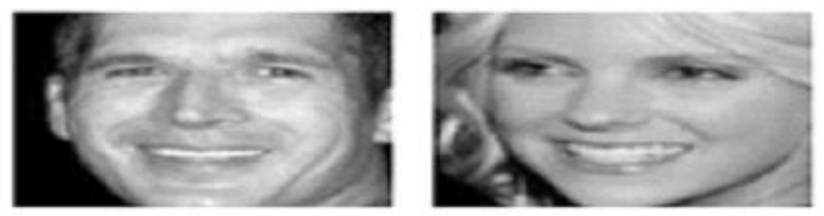

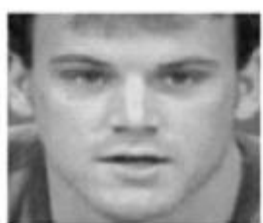

(a)
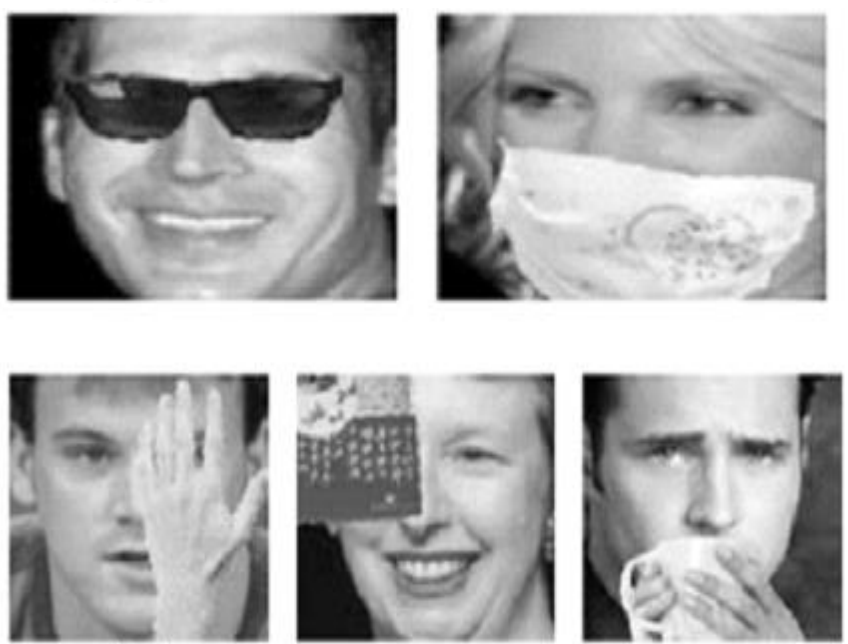

(b)
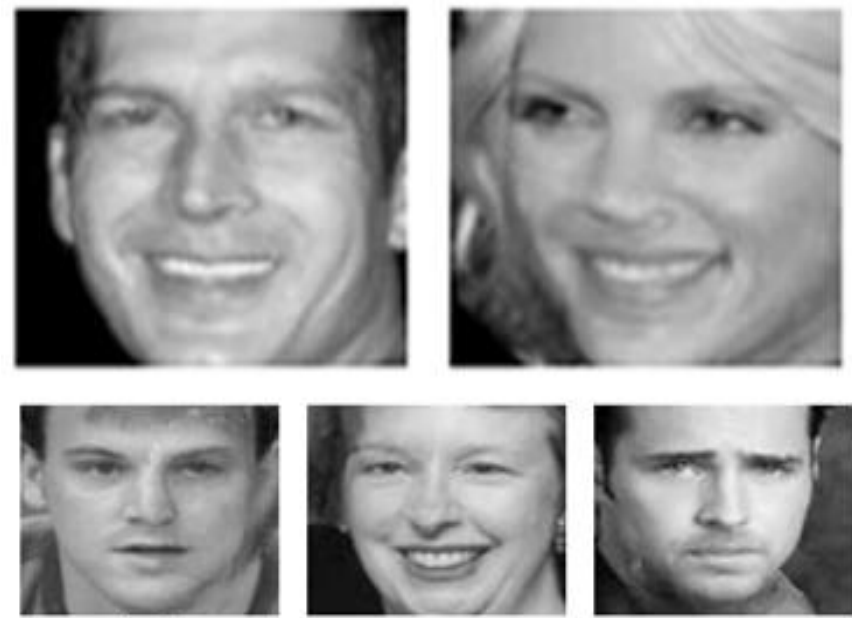

(c)

Figure 2 (a)Occlusion-free human faces(Original); (b) Occluded human faces; (c)Regained human faces by our suggested method.

2) Face Recognition: This experiment does the analysis of face confirmation on the appearances recouped by de-impediment strategies to additionally examine the capacity of our model in perceiving blocked countenances. Initially distillate include vectors for a couple of human face images (first is an impediment free human face, and another is a recuperated face or a blocked face) and register a comparability between 
element vectors utilizing Euclidean separation between informational index input images to choose if the pair of countenances from a similar subject. KNN-NMC is employed to remove face highlights in the analysis.

Note that consolidating with the impediment recognition essentially decreases the blunder rate contrasted and recuperating faces without utilizing impediment identification. Like the perceptions made in the subjective examination, impediment expulsion for quarter or right/left $50 \%$ of the human face to improve better presentation of blocked human face identification in the light of the fact that the appearances of blocked facial segments can be anticipated by the nonblocked parts by using facial balance. Notwithstanding, recouped human faces for lower faces or upper faces actually accomplish lower mistake rate contrasted and impeded human faces, that demonstrate that our prototype can learn relations among upper and lower faces and concentrate discriminative highlights from non-blocked (lower) upper appearances to recuperate impeded (upper)lower faces.
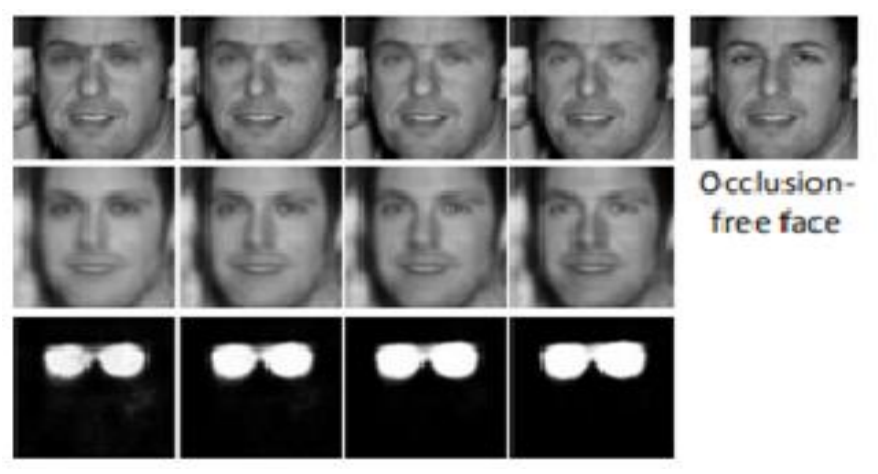

Occlusionfree face
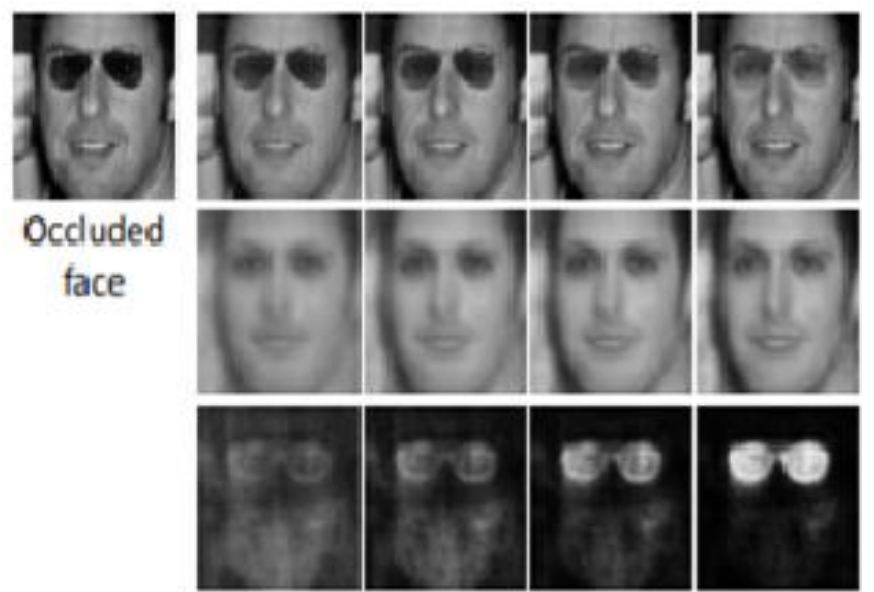
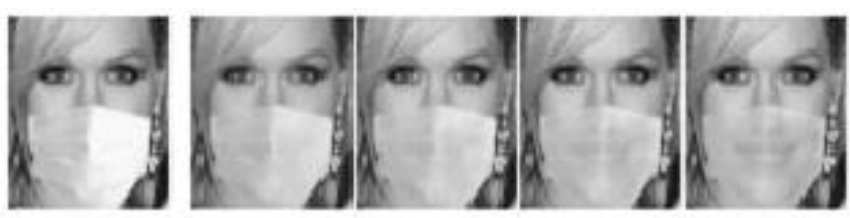

\section{Occluded \\ face}
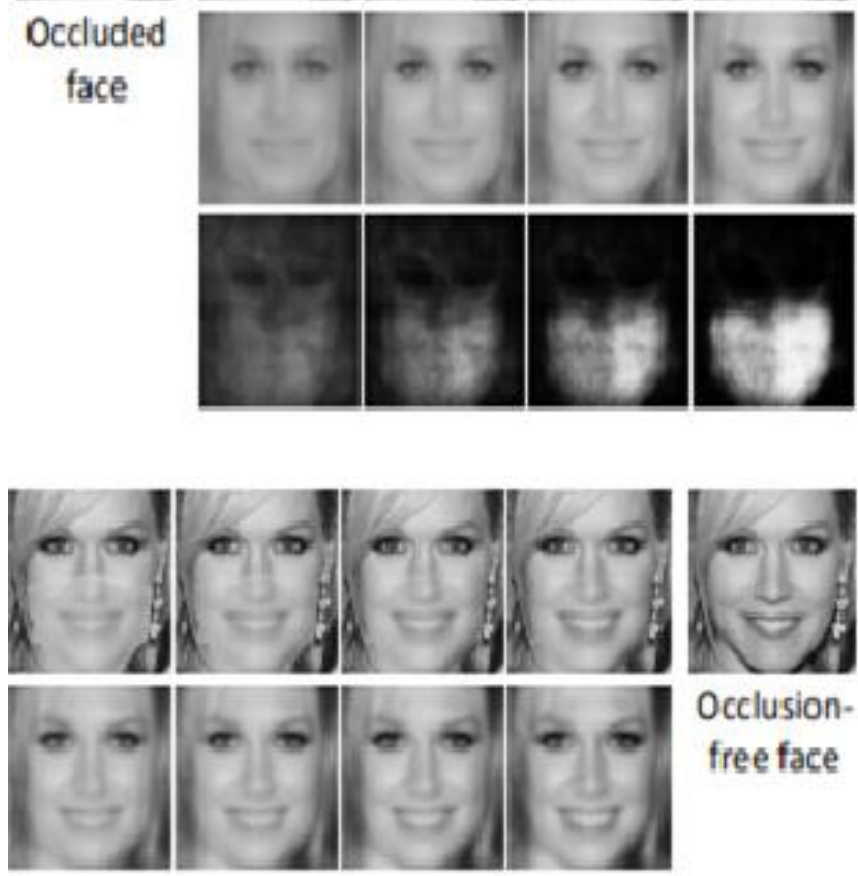

Occlusion-

free face

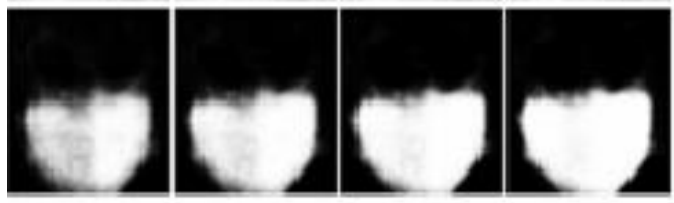

Figure 3 Occlusion removal process of Robust K-NNC \& NMC.

In this experiments arbitrarily tested 10,000 sets (positive sets of 5000 and negative sets of 5000) of appearances have been considered for the analysis. The Equal Error Rates(EER) found the middle value of on a wide range of impediment are recorded in Table 1 . Checking the result indicates that our prototype beats different strategies; moreover, it can be summed up to genuine blocked face information. The center columns show yields of Teacher stream. The main line shows yields of the Robust K-NNC\&NMC with both face recreation and impediment recognition parts. The last column shows yields from understudy stream just face recreation (without impediment detection). The third line shows the discovery consequences of impediment. 

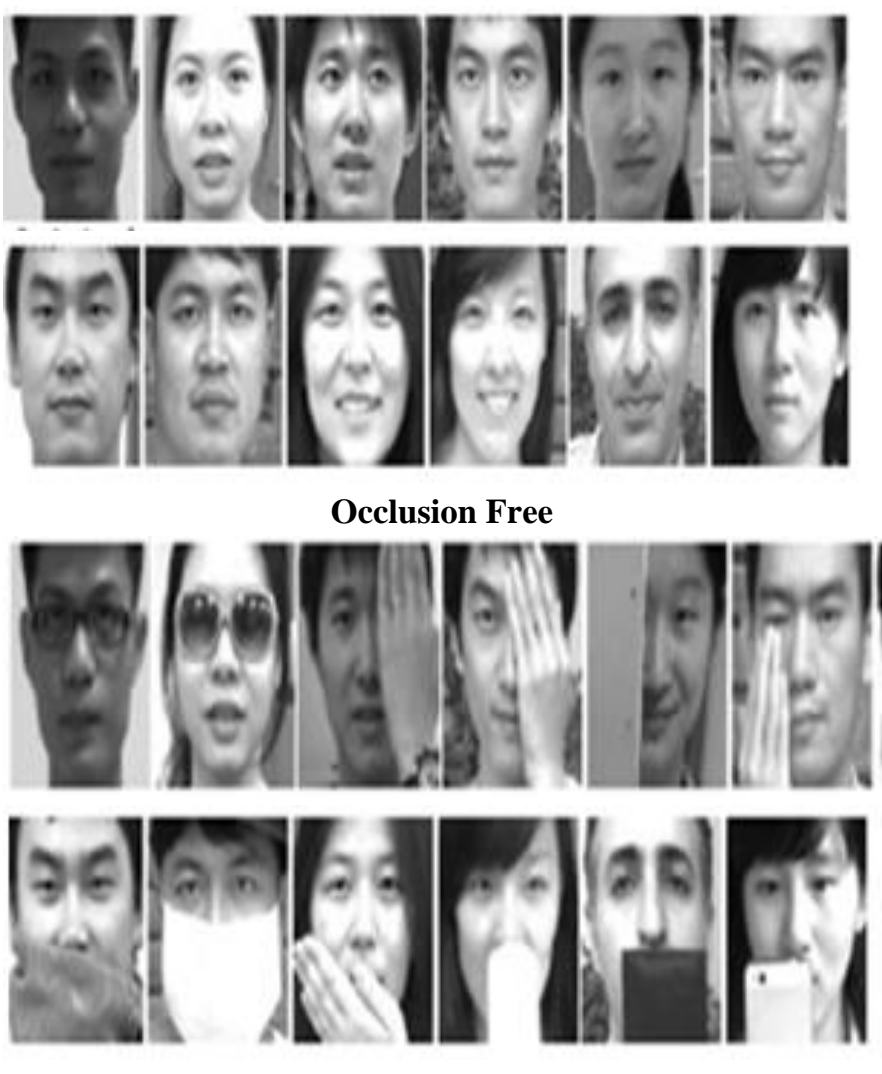

Occluded

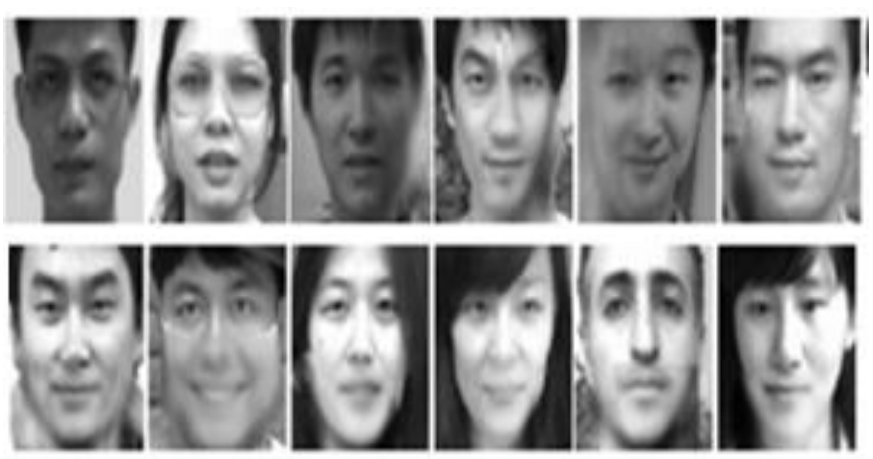

K-NNC \& NMC

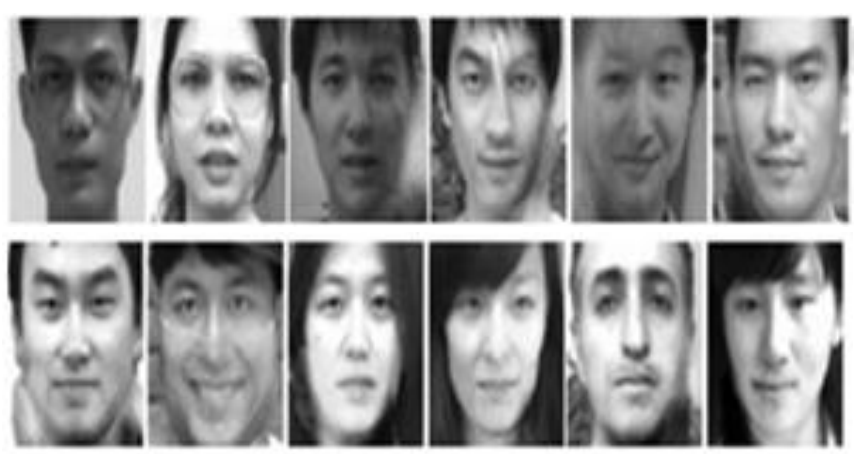

Robust K-NNC \& NMC

Figure 4 Occlusion free faces (normal faces) are represented in the first row, occluded faces are represented in the second row, and the remaining rows display the outcomes of Robust KNNC \& NMC.
The Teacher stream of the analyses done on eye and mouth locales of a human face by utilizing Region based element extraction plot is organized in Table 2.

(Anger abbreviated as AN, similarly Disgust as DI, Fear as FE, Happy as HA, Sad as SA and Surprise as SU)

This is cultivated for all subjects; so each subject is used for testing only a solitary time.

\begin{tabular}{|c|c|c|c|c|}
\hline \multicolumn{2}{|c|}{ Types of occlusion } & \multirow{2}{*}{$\begin{array}{l}\text { PCA } \\
12.4 \%\end{array}$} & LPP & \multirow{2}{*}{$\begin{array}{l}\text { UDP } \\
19.6 \%\end{array}$} \\
\hline $\begin{array}{l}\text { Quarter of } \\
\text { face }\end{array}$ & Hand & & $18.6 \%$ & \\
\hline $\begin{array}{l}\text { Left/right half } \\
\text { of face }\end{array}$ & Hand & $18.6 \%$ & $24.7 \%$ & $22.6 \%$ \\
\hline Upper face & glasses & $11.8 \%$ & $17.4 \%$ & $14.4 \%$ \\
\hline Upper face & $\begin{array}{l}\text { sunglasse } \\
\mathrm{S}\end{array}$ & $20.7 \%$ & $18.9 \%$ & $22.3 \%$ \\
\hline Upper face & $\begin{array}{l}\text { Eye } \\
\text { mask }\end{array}$ & $33.3 \%$ & $36.2 \%$ & $35.4 \%$ \\
\hline Lower face & mask & $21.4 \%$ & $24.7 \%$ & $25.7 \%$ \\
\hline Lower face & phone & $14.2 \%$ & $18.5 \%$ & $17.5 \%$ \\
\hline Lower face & Cup & $12.7 \%$ & $15.2 \%$ & $16.2 \%$ \\
\hline Lower face & scarf & $17.3 \%$ & $18.2 \%$ & $19.1 \%$ \\
\hline \multirow{2}{*}{\multicolumn{2}{|c|}{ Types of occlusion }} & \multicolumn{3}{|c|}{$\begin{array}{l}\text { Proposed method K-NNC, } \\
\text { NMC }\end{array}$} \\
\hline & & \multicolumn{2}{|c|}{\begin{tabular}{l|l}
$\begin{array}{l}\text { Normal } \\
\text { face }\end{array}$ & No \\
fac
\end{tabular}} & $\begin{array}{l}\text { Normal } \\
\text { face }\end{array}$ \\
\hline Quarter of face & Hand & $8.5 \%$ & \multicolumn{2}{|c|}{$11.3 \%$} \\
\hline $\begin{array}{l}\text { Left/right half } \\
\text { of face }\end{array}$ & Hand & $15.6 \%$ & \multicolumn{2}{|c|}{$16.7 \%$} \\
\hline Upper face & glasses & $8.2 \%$ & \multicolumn{2}{|c|}{$8.6 \%$} \\
\hline Upper face & $\begin{array}{l}\text { sunglass } \\
\text { es }\end{array}$ & $17.6 \%$ & \multicolumn{2}{|c|}{$17.9 \%$} \\
\hline Upper face & $\begin{array}{l}\text { Eye } \\
\text { mask }\end{array}$ & $28.9 \%$ & \multicolumn{2}{|c|}{$31.5 \%$} \\
\hline Lower face & mask & $15.7 \%$ & \multicolumn{2}{|c|}{$15.9 \%$} \\
\hline Lower face & phone & $12.3 \%$ & \multicolumn{2}{|c|}{$12.8 \%$} \\
\hline Lower face & Cup & $8.9 \%$ & \multicolumn{2}{|c|}{$9.1 \%$} \\
\hline Lower face & scarf & $13.4 \%$ & \multicolumn{2}{|c|}{$13.9 \%$} \\
\hline
\end{tabular}

Table 1. EER (Equal Error Rates) Of Occluded Face Recognition for LFW Datasets

This evaluation plot guarantees that images from one subject are not in the testing and handling set all the while, so the classifier cannot learn "inside class" relations. The ordinary system to develop subspaces, for instance in PCA, is to plan 
genuinely on crude pixel power of the images. Our story subspace depiction yields imperative improvement in recognition since we input remembered images as shown in Figure 3 instead of unrefined pixel power for subspace illustrating. Examples of top 3 Eigen faces from K-NNC-NMC using both rough pixel power and normalized cosine partition assessment to enlist equivalence structure.

\begin{tabular}{|l|l|l|l|}
\hline $\begin{array}{l}\text { Region based Feature } \\
\text { Extraction }\end{array}$ & AN & DI & FE \\
\hline Eye Region & 35.5 & 76.2 & 32.6 \\
\hline Mouth Region & 73.2 & 84.6 & 86.1 \\
\hline Nose Region & 23.6 & 72.8 & 26.6 \\
\hline Whole Face & 57.4 & 91.2 & 47.4 \\
\hline $\begin{array}{l}\text { Region based Feature } \\
\text { Extraction }\end{array}$ & HA & SA & SU \\
\hline Eye Region & 69.7 & 45.6 & 87.4 \\
\hline Mouth Region & 93.2 & 82.6 & 86.4 \\
\hline Nose Region & 52.6 & 36.1 & 40.4 \\
\hline Whole Face & 86.4 & 57.4 & 65.4 \\
\hline
\end{tabular}

Table 2 Similarity of identification rates gained by employing Region based feature extraction scheme on different facial regions (\%)

Regarding Table 2, recognition exactness is discovered to be poor for the greater part of the articulations (aside from nauseate) when just nose locale is associated with articulation recognition. Be that as it may, a huge improvement has been found in the identification pace of outrage, dread, miserable articulations whether just mouth district included when contrasted with tests done on the entire facial area. Likewise, the recognition precision is discovered to be useful for exploring Region based elements from the entire face locale. The after effects of the analyses done indicate the connection between facial locales and plausibility of event of articulations. It might be considered that impediment regarding nose and eye area negotiably affects the exhibition of proposed System.

\section{Conclusion}

In this manuscript is proposed Robust KNNC\&NMC to address the issue of Occluded face and appearance in face recognition. The proposed model is demonstrated to have the option to viably recuperate blocked facial parts dynamically. The recommended task is an endeavor made about individual commitment of facial areas about outward appearance identification. The three unmistakable features from the face districts are Eye, Mouth and Nose have been considered for experimentation. Contingent on the demeanor important to be distinguished, choice of facial locale for including extraction assumes a conspicuous job. After effects of the investigations completed on Low-Resolution Face Verification on LFW information base uncovers that there is critical commitment from mouth locale towards conceptual and strong component extraction concerning appearance grouping.

\section{References}

[1] Cheng, B., Liu, D., Wang, Z., Zhang, H., \& Huang, T. S. (2018, January). Visual recognition in very low-quality settings: Delving into the power of pretraining. In 32nd AAAI Conference on Artificial Intelligence, AAAI 2018 (pp. 8065-8066). AAAI Press.

[2] Mudunuri, S. P., Venkataramanan, S., \& Biswas, S. (2018). Dictionary alignment with re-ranking for low-resolution NIR-VIS face recognition. IEEE Transactions on Information Forensics and Security, 14(4), 886-896.

[3] Li, P., Prieto, L., Mery, D., \& Flynn, P. J. (2019). On low-resolution face recognition in the wild: Comparisons and new techniques. IEEE Transactions on Information Forensics and Security, 14(8), 2000-2012.

[4] Singh, M., Nagpal, S., Vatsa, M., Singh, R., \& Majumdar, A. (2018). Identity aware synthesis for cross resolution face recognition. In Proceedings of the IEEE Conference on Computer Vision and Pattern Recognition Workshops (pp. 479-488).

[5] Chen, G., Choi, W., Yu, X., Han, T., \& Chandraker, M. (2017). Learning efficient object detection models with knowledge distillation. In Advances in Neural Information Processing Systems (pp. 742751).

[6] Chen, Y., Wang, N., \& Zhang, Z. (2017). Darkrank: Accelerating deep metric learning via cross sample similarities transfer. arXiv preprint arXiv: 1707.01220 .

[7] Chen, T., Lin, L., Zuo, W., Luo, X., \& Zhang, L. (2017). Learning a wavelet-like auto-encoder to accelerate deep neural networks. arXiv preprint arXiv: 1712.07493.

[8] Zhou, G., Fan, Y., Cui, R., Bian, W., Zhu, X., \& Gai, K. (2017). Rocket launching: A universal and efficient framework for training well-performing light net. arXiv preprint arXiv:1708.04106.

[9] Lopez-Paz, D., Bottou, L., Schölkopf, B., \& Vapnik, V. (2015). Unifying distillation and privileged information. arXiv preprint arXiv: 1511.03643 .

[10] Su, J. C., \& Maji, S. (2016). Adapting models to signal degradation using distillation. arXiv preprint arXiv: 1604.00433 .

[11] BIOID. Bioid face database. http://www.bioid.com/support/download s/software/bioid-face-database.html. 
[12] Blanz, V., \& Vetter, T. (1999, July). A morphable model for the synthesis of 3D faces. In Proceedings of the 26th annual conference on Computer graphics and interactive techniques (pp. 187-194).

[13] Blanz, V., \& Vetter, T. (2003). Face recognition based on fitting a $3 \mathrm{~d}$ morphable model. IEEE Transactions on pattern analysis and machine intelligence, 25(9), 1063-1074.

[14] Bledsoe, W. W. (1964). The model method in facial recognition, panoramic research inc., Palo Alto. CA, Technical Report, Technical Report PRI: 15.

[15] Bledsoe, W. W. (1966). Man-machine facial recognition. Rep. PRi, 22.

[16] Bledsoe, W. W. (1966). Some results on multicategory pattern recognition. Journal of the ACM (JACM), 13(2), 304-316.

[17] Bledsoe, W. W. (1968). Semiautomatic facial recognition. Technical report sri project 6693.

[18] Bledsoe, W. W., \& Chan, H. (1965). A manmachine facial recognition system-some preliminary results. Panoramic Research, Inc, Palo Alto, California., Technical Report PRI A, 19, 1965.

[19] Bronstein, A. M., Bronstein, M. M., Kimmel, R., \& Spira, A. (2003). 3D face recognition without facial surface reconstruction (No. CS Technion report CIS-2003-05). Computer Science Department, Technion.

[20] [20] Brunelli, R., \& Poggio, T. (1993). Face recognition: Features versus templates. IEEE transactions on pattern analysis and machine intelligence, 15(10), 1042-1052.

[21] Bruner, J. S., \& Tagiuri, R. (1954). The perception of people (Vol. 2). In Handbook of Social Psychology, 634-654. 